\title{
Distribuição espaço-temporal de ocorrências de dengue em Três Corações, Minas Gerais, utilizando processos pontuais
}

\author{
Spatio-temporal distribution of dengue occurrences in \\ Três Corações, Minas Gerais, using point processes
}

\author{
Rodrigo Ferreira de Abreu ${ }^{1}$, Rafael Agostinho Ferreira ${ }^{2}$, \\ João Domingos Scalon ${ }^{3}$
}

Resumo

\begin{abstract}
Objetivo: detectar a presença de agrupamentos espaço-temporais dos casos de dengue em Três Corações, Minas Gerais, Brasil, utilizando informações da localização e do tempo de cada ocorrência e a série histórica da precipitação pluviométrica do período de estudo. Métodos: o método Kernel foi utilizado para estimar a intensidade dos casos, enquanto a função $K$ espaço-temporal e o método de varredura foram utilizados para detectar o padrão e identificar agrupamentos, respectivamente. Resultados: a partir dos 2.818 casos observados, verificou-se que a maior parte desses ocorreu no final dos períodos chuvosos. Também foi detectada a presença de agrupamentos de casos, principalmente na Região Central da cidade. Uma razão para a formação de agrupamentos pode ser devido à maior densidade populacional das regiões afetadas. Conclusão: os resultados mostram que indivíduos que moram em regiões densamente povoadas são mais propensos a contrair dengue. Os métodos estatísticos utilizados permitiram caracterizar a distribuição espaço-temporal dos casos de dengue e também podem ser utilizados para analisar outras doenças endêmicas ou pandêmicas, o que pode contribuir para as políticas de prevenção e combate à proliferação dessas doenças.
\end{abstract}

Palavras-chave: Epidemiologia. Aedes aegypti. Métodos estatísticos. Pluviosidade.

\begin{abstract}
Objective: detect the presence of space-time clusters of dengue cases in Três Corações, Minas Gerais, Brazil, using information on the location and time of each occurrence and the historical series of rainfall in the study period. Methods: the Kernel method was used to estimate the intensity of the cases, while the space-time K-function and the scan method were used to detect the pattern and identify clusters, respectively. Results: from the 2,818 observed cases, it was found that most of these occurred in the end of rainy periods. The presence of clusters of cases was also detected, mainly, in the central region of the city. One reason for the formation of clusters may be due to the higher
\end{abstract}

\footnotetext{
${ }^{1}$ Doutorando em Estatística e Experimentação Agropecuária na Universidade Federal de Lavras (UFLA), Lavras, Minas Gerais, Brasil. Professor Substituto no Departamento de Estatística da Universidade Federal de Lavras, Lavras, Minas Gerais, Brasil. E-mail: abreurf.est@gmail.com

${ }^{2}$ Doutorando em Estatística e Experimentação Agropecuária na Universidade Federal de Lavras, Lavras, Minas Gerais, Brasil.

${ }^{3}$ Doutorado em Probabilidade e Estatística pela University of Sheffield, Sheffield, Inglaterra. Professor Titular do Departamento de Estatística da Universidade Federal de Lavras, Lavras, Minas Gerais, Brasil.
} 
population density of the affected regions. Conclusion: the results show that individuals who live in densely populated regions are more likely to get dengue. The statistical methods used allowed to characterize the spatio-temporal distribution of dengue cases and, they can also be used to analyze other endemic or pandemic diseases, which can contribute to policies to prevent and combat the proliferation of these diseases.

Keywords: Epidemiology. Aedes aegypti. Statistical methods. Rainfall.

\section{Introdução}

Desde a década de 1980, a dengue tem sido uma doença infecciosa que tem causado grandes transtornos no Brasil, onde milhões de pessoas já foram infectadas ${ }^{(1)}$ e muitas vieram a óbito em função dela. ${ }^{(2)}$ A localização do Brasil na zona tropical com clima quente e úmido é um fator que contribui para a propagação da dengue. Além disso, outros fatores que concorrem para a formação das epidemias de dengue nos países tropicais e subtropicais incluem a proliferação do mosquito transmissor, infraestrutura inadequada, e intensa e desordenada urbanização. Há que se destacar também a resistência do mosquito quanto às formas de controle. No caso do Brasil, a pressão pelo uso intensivo de inseticidas, como themephos e deltametrina, acarretou a seleção de indivíduos resistentes aos mesmos. ${ }^{(3)}$

De acordo com a Organização Mundial da Saúde, ${ }^{(4)}$ a dengue é uma doença causada por um vírus (DEN) que compreende quatro sorotipos (DEN-1, DEN-2, DEN-3 e DEN-4) que apresentam características genéticas distintas. Os vários sorotipos do vírus da dengue são transmitidos aos seres humanos através das picadas de mosquitos Aedes infectados, sendo o Aedes aegypti o principal vetor. Vale ressaltar que este agente transmissor é também responsável pela disseminação de outros arbovírus, tais como chikungunya, zika e febre amarela. ${ }^{(5)}$

Em relação à transmissão da doença, é importante destacar que apenas a fêmea do mosquito transmite o vírus pela picada, uma vez que utiliza o sangue para amadurecer seus ovos. Durante a picada, o mosquito libera saliva contendo substâncias anticoagulantes e analgésicas, fazendo com que não seja notado e, consequentemente, permite sugar a maior quantidade de sangue possível. Neste processo, o vírus é injetado na corrente sanguínea junto com a saliva do mosquito. ${ }^{(6-7)}$

A maior concentração (80\%) dos focos do mosquito está localizada nas residências e a maioria das fêmeas do $A$. aegypti podem passar a vida dentro ou em torno das casas onde emergem como adultas. Uma fêmea pode voar entre 50 e 500 metros e pode picar por toda sua vida adulta, que dura aproximadamente 30 dias. $^{(8-10)}$ Considerando períodos recentes como de 2016 a 2019, dados do Ministério da Saúde do Brasil ${ }^{(11)}$ mostram que as ocorrências no País têm apresentado números expressivos. No ano de 2016 o número de casos de dengue foi de mais de 1,5 milhão de casos. Em 2017 e 2018 houve uma queda no número de casos: foram registrados cerca de 239 mil e 266 mil casos prováveis da doença, respectivamente. $\mathrm{O}$ número de casos de dengue registrados no Brasil em 2019 foi o segundo mais alto da série histórica. Os dados apontam 1,527 milhão de notificações, concentradas principalmente nas regiões Sudeste e Centro-Oeste. Quase dois terços dessas ocorrências foram nos estados de São Paulo, Minas Gerais e Espírito Santo.

Devido à expressividade da dengue no Brasil, é necessário que haja sempre uma busca por mecanismos que possam contribuir para a compreensão da forma como o mosquito transmissor se propaga e, com isso, auxiliar no monitoramento para estratégias de controle específicas da doença. Deste modo, metodologias estatísticas podem corroborar para a compreensão da dinâmica da doença com base em dados observacionais. Assim, diversos estudos com diferentes abordagens foram e continuam a ser desenvolvidos. Dentre eles há os que se dedicam ao estudo da dispersão espacial e/ou temporal da ocorrência dessa enfermidade. 
Por exemplo, é usual agrupar dados de ocorrências de dengue, por áreas ou setores censitários, e avaliar se existe algum grau de relacionamento entre áreas próximas. A análise estatística pode ser realizada considerando espaço e tempo separadamente ou conjuntamente. ${ }^{(12-15)}$ Pode-se também incluir nas análises informações ambientais ${ }^{(16)}$ com o objetivo de verificar se existe associação entre a dengue e fatores climáticos.

O objetivo deste trabalho é analisar a distribuição espaço-temporal de casos de dengue na cidade de Três Corações, Minas Gerais, Brasil, entre os anos de 2010 e 2015, utilizando como ferramentas métodos da teoria de processos pontuais, na área de estudo da estatística espacial, para tentar entender os mecanismos existentes no processo, identificando a presença de agrupamentos de casos na região de estudo, considerando as informações da localização e do tempo de cada ocorrência e a série histórica de precipitação pluviométrica do período de estudo. É importante enfatizar que, por mais que o foco do trabalho seja a dengue, a caracterização da distribuição espaço-temporal de casos de outras doenças como chikungunya, febre amarela urbana e Covid-19, entre outras, também pode ser realizada utilizando os métodos apresentados neste trabalho.

\section{Material e Métodos}

Local de estudo

Para desenvolver este trabalho foram utilizados métodos relacionados à teoria de processos pontuais espaço-temporais, aplicados em dados constituídos pelas informações espaciais e temporais das ocorrências de dengue na cidade de Três Corações, no período de $1^{\circ}$ de janeiro de 2010 a 31 de dezembro de 2015.

A cidade de Três Corações situa-se na Região Sul do estado de Minas Gerais na latitude $21^{\circ} 42^{\prime} 00^{\prime}$ S e longitude $45^{\circ} 15^{\prime} 30^{\prime \prime} \mathrm{W}$, a uma altitude de 839 metros acima do nível do mar e a $292 \mathrm{~km}$ ao sul da capital Belo Horizonte. ${ }^{(17)} \mathrm{De}$ acordo com o Instituto Brasileiro de Geografia e
Estatística (IBGE), ${ }^{(18)}$ a área do município é de $828,038 \mathrm{~km}^{2}$ e foi registrado no censo de $2010 \mathrm{uma}$ população de 72.765 habitantes, sendo $90,5 \%$ de residentes na área urbana. A população estimada para o ano de 2019 foi de 79.482 habitantes.

O clima é classificado como temperado úmido com inverno seco e verão quente, com temperatura média de $20,2{ }^{\circ} \mathrm{C}$ e pluviosidade média anual de $1.401 \mathrm{~mm}$. O período com maior volume de chuvas ocorre de outubro a março, sendo dezembro o mês com o maior acumulado médio de precipitação (270 mm). Já o mês com menor precipitação é julho, com $15 \mathrm{~mm}$. Normalmente, fevereiro é o mês mais quente do ano, com média de $23,2{ }^{\circ} \mathrm{C}$ e com uma temperatura média de $16,2{ }^{\circ} \mathrm{C}$; junho é o mês mais frio. A diferença de precipitação entre o mês mais seco e o mais chuvoso é de $255 \mathrm{~mm}$, e as temperaturas médias variam em $7^{\circ} \mathrm{C} .{ }^{(19)}$

O conjunto de dados

O conjunto de dados analisados nesta pesquisa foi obtido junto à Vigilância Epidemiológica da cidade de Três Corações. A localização dos casos de dengue foi composta pelo endereço residencial das pessoas diagnosticadas e a data da ocorrência de cada evento corresponde ao momento da notificação no serviço de saúde.

A preparação dos dados para a análise constituiu-se, basicamente, da transformação dos endereços residenciais em pontos georreferenciados na forma UTM (Universal Transversa de Mercator). Para isso, utilizou-se a base de dados do Google Earth $^{(20)}$ e as bibliotecas RgoogleMaps ${ }^{(21)}$ e $s p^{(22)}$ do software R (R Core Team). ${ }^{(23)}$

No arquivo de dados inicial, o número total de ocorrências foi de 3.456 , em que o número de casos para cada ano do período 2010-2015 foi respectivamente de 381, 471, 55, 58, 1.056 e 1.435 . Contudo, para o trabalho foram desconsiderados os casos em que o ponto correspondente ao endereço ocorreu fora da área urbana, assim como os casos em que as informações de endereço não eram suficientes, ou estavam incorretas, o que impossibilitou a localização das coordenadas. 
A partir desse primeiro tratamento dos dados, o conjunto de dados analisado no trabalho passou a ter então, respectivamente, ao período 2010-2015, quantidades de casos iguais a 304, 397, 51, 50, 854 e 1.162, totalizando 2.818 ocorrências de dengue.

A série histórica da precipitação pluviométrica para o período de estudo foi obtida a partir das bases de dados do Sistema Integrado de Dados Ambientais (SINDA) - do Instituto Nacional de Pesquisas Espaciais (INPE), disponível em http:// sinda.crn.inpe.br/PCD/SITE/novo/site/historico/ index.php, e do sistema Hidroweb da Agência Nacional de Águas (ANA), disponível em https:// www.snirh.gov.br/hidroweb/serieshistoricas. A série obtida, no entanto, possui informações faltantes para os meses de maio de 2010, abril a julho de 2013, julho e agosto de 2014 e outubro a dezembro de 2015.

\section{Análise de processos pontuais}

Um processo pontual espaço-temporal é um processo estocástico que gera um conjunto contável de pontos $\left\{\left(\mathbf{s}_{i}, t_{i}\right): i=1, \ldots, n\right\}$, em que $t_{i} \in$ $\mathbb{R}^{+}$e $\mathbf{s}_{i} \in S \subset \mathbb{R}^{2}$. Os processos pontuais podem ser estudados em termos de quantidades denominadas por efeitos de primeira e/ou segunda ordem.

Efeitos de primeira ordem são ditos globais ou de larga escala, e correspondem a variações no valor médio do processo. Nesse caso, o interesse está no número de eventos por unidade de área e/ ou tempo, dado pela intensidade $\lambda$ do processo, que pode ser homogênea ou não homogênea, isto é, é constante em toda parte ou pode variar, exibindo um comportamento de tendência.

A estimação da intensidade $\lambda$ em um ponto $\mathbf{s}_{0}$ qualquer de uma região $S$ pode ser feita a partir de estimadores baseados em funções de $\mathrm{Kernel}^{(24)}$ da forma:

$$
\widehat{\lambda}=\frac{1}{|S| \boldsymbol{h}} \sum_{i=1}^{n} k\left(\frac{\boldsymbol{s}_{i}-\boldsymbol{s}_{0}}{\boldsymbol{h}}\right),
$$

em que $|S|$ é a área de $S$, $h$ é um parâmetro chamado largura de banda e $k$ é uma função Kernel que satisfaz $k(x) \mathrm{d} x=1$ e $x k(x) \mathrm{d} x=0$.
Os efeitos de segunda ordem são locais ou de pequena escala e representam a dependência no processo, em função de uma estrutura de correlação espaço-temporal. Na análise dos efeitos de segunda ordem procura-se responder se os eventos estão mais próximos ou mais distantes entre si, exibindo algum grau de dependência maior do que seria esperado no caso de completa aleatoriedade espaço-temporal (CAET), ou seja, quando a distribuição das ocorrências não exibe nenhum tipo de padrão.

$\mathrm{Na}$ CAET, os eventos distribuem-se uniformemente e independentemente ao longo da região e/ou período de estudo e a sua manifestação matemática é o chamado processo de Poisson homogêneo. Esse é um processo sem qualquer estrutura espaço-temporal e serve como hipótese nula para muitas investigações estatísticas em padrões pontuais.

Os padrões de pontos observados são testados inicialmente contra a hipótese de CAET e se essa hipótese for rejeitada, então o pesquisador geralmente segue com análises mais específicas para investigar a natureza do padrão pontual.

$\mathrm{Na}$ literatura existem diversos métodos para investigar o padrão de ocorrência de eventos que tem uma distribuição espaço-temporal. A função $K^{(25)}$ espacial é uma medida de dependência espacial, que utiliza as propriedades de segunda ordem do processo pontual para identificar o padrão de distribuição dos eventos para diferentes escalas de distâncias.

A função $K$ espaço-temporal é definida ${ }^{(25)}$ por $K(u, v)=\lambda^{-1} E[N(u, v)]$, em que $E[N(u, v)]$ é o número médio esperado de eventos encontrados dentro de uma distância $u$ e tempo $v$ e $\lambda$ é a intensidade homogênea do processo pontual. Sob a hipótese de CAET, ou seja, quando não existe uma estrutura de correlação espaço-temporal entre as ocorrências, pode-se mostrar que a função $K$ teórica é dada por $K(u, v)=\pi u^{2} v$.

Um estimador para a função $K$ homogênea espaço-temporal com base em dados que fornecem a localização dos eventos $\left\{\left(\mathbf{s}_{i}, t_{i}\right): i=1, \ldots, n\right\}$ numa região espaço-temporal é dado por Diggle et al. ${ }^{(25)}$ 
e pode ser calculado utilizando a biblioteca $\operatorname{stpp}^{(26)}$ Para o procedimento de análise com a função $K$, compara-se o resultado da função $K$ estimada $(\widehat{K}(u, v))$ com o esperado pelo processo de Poisson homogêneo $\left(\pi u^{2} v\right)$ através da diferença $\widehat{K}(u, v)$ $\pi u^{2} v$.

Um resultado apresentando excesso de valores positivos significa que o número observado de eventos é maior do que o esperado pela CAET, indicando, portanto, a presença de agrupamentos espaço-temporais em uma separação espacial e temporal inferiores a $u$ e $v$, respectivamente. Já um excesso de valores negativos significa que o número de eventos observados é menor do que o esperado pela CAET, indicando que existe repulsão entre os eventos. Caso os valores sejam relativamente próximos de zero, não se rejeita a hipótese de que a configuração espaço-temporal exibe CAET.

Uma estatística de teste para verificar a hipótese de CAET é dada ${ }^{(27)}$ por:

$$
D=\int_{0}^{v} \int_{0}^{u} \frac{\left(\widehat{K}(u, v)-\pi u^{2} v\right)^{2}}{\pi u^{2} v} .
$$

A estatística de teste $D$ não apresenta distribuição amostral conhecida. Assim, utilizamse simulações de Monte Carlo, cujo procedimento consiste em obter a distribuição empírica das estatísticas $D$ calculadas a partir de $N$ simulações independentes de um processo de Poisson espaçotemporal homogêneo com intensidade constante $\lambda$, dada pelo número médio de eventos por unidade de área e unidade de tempo, condicionado para ter $n$ eventos na região de estudo. A partir do nível de significância determinado para o teste, verificase então o valor-p obtido como a proporção de estatísticas de teste $D$ calculadas a partir de processos simulados iguais ou superiores à estatística observada. Deste modo têm-se:

$$
\text { valor-p }=\frac{R}{N+1}
$$

em que $R$ é a classificação da estatística $D$ observada entre as $D_{\mathrm{N}+1}$ estatísticas ordenadas. A partir desse procedimento, o menor valor-p possível de se obter é então $1 /(N+1)$.
Caso o interesse da análise seja encontrar agrupamentos em sub-regiões geográficas e intervalos de tempos específicos, pode utilizar algum método estatístico de varredura. ${ }^{(28)} \mathrm{Um}$ desses métodos de varredura espaço-temporal utiliza milhares de cilindros sobrepostos, cujo raio e altura variam de tamanho, para definir a janela de varredura, sendo cada cilindro um possível candidato a um agrupamento.

Esse método não requer dados da população em risco; assim, o número de casos esperado é calculado usando apenas os casos observados, e pode ser utilizado tanto para dados agregados por áreas como para coordenadas únicas, que é o caso deste trabalho.

Considerando um cilindro $A$ qualquer dentro da região de estudo, $\mathrm{c}_{\mathrm{A}} \mathrm{o}$ número observado de casos em $A$ e $C$ o número total de casos, tem-se que $\mathrm{c}_{\mathrm{A}}$ segue uma distribuição, aproximadamente, de Poisson com média $\mu_{\mathrm{A}}$. A partir disso, se utiliza a razão de verossimilhança generalizada de Poisson $k$, como medida da evidência de que o cilindro $A$ contém um agrupamento dada por:

$$
k_{k}(A)=\left(\frac{c_{A}}{\mu_{A}}\right)^{c_{A}}\left(\frac{C-c_{A}}{C-\mu_{A}}\right)^{c_{A}} .
$$

Entre os muitos cilindros avaliados, aquele com o máximo de $k(A)$ constitui o aglomerado espaço-tempo de casos que é menos provável de ser uma ocorrência completamente aleatória no espaço e no tempo e, portanto, é o candidato para um verdadeiro agrupamento de eventos. Para verificar se esse cilindro exibe um agrupamento espaço-temporal estatisticamente significante, utiliza-se o procedimento de Monte Carlo abordado anteriormente.

Para essa análise de varredura foi utilizando o software SaTScan ${ }^{\mathrm{TM}}$. $^{(29)}$

Por utilizar apenas dados secundários agregados, sem a identificação de qualquer indivíduo, o trabalho atendeu às considerações éticas previstas nas resoluções do Conselho Nacional de Saúde (CNS) n ${ }^{\circ} 466$, de 12 de dezembro de 2012, e n ${ }^{\circ} 510$, de 7 de abril de 2016, estando isento de avaliação por comitê de ética em pesquisa. 


\section{Resultados}

Fazendo um refinamento mensal dos 2.818 casos de dengue analisados, pode-se observar, a partir da Figura 1, um padrão de sazonalidade no período em estudo, em que os intervalos de maiores ocorrências de casos de dengue ocorrem entre os meses de março a maio, com destaque para o mês de abril, quando acontece o pico das ocorrências.

Figura 1 - Distribuição mensal das ocorrências de dengue em Três Corações, Minas Gerais, entre os anos de 2010 e 2015.

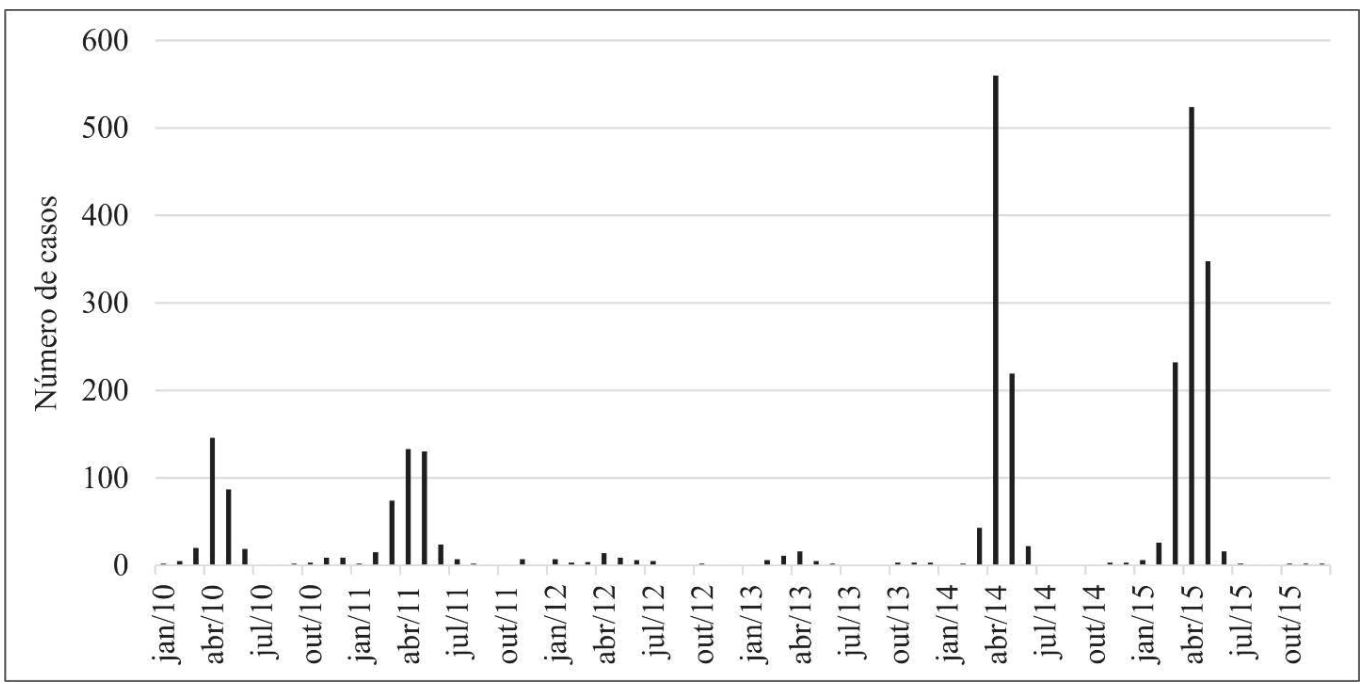

Fonte: Autores

Combinando essa informação de casos de dengue, com a série histórica de precipitação pluviométrica no mesmo período, pode-se notar a partir da Figura 2, que nos anos em que ocorreram surtos, o número de casos começou a aumentar no final do período chuvoso até atingir o pico, e de estudo.

Figura 2 - Comparativo mensal entre a quantidade de chuvas e número de casos de dengue em Três Corações, Minas Gerais, entre os anos de 2010 e 2015.

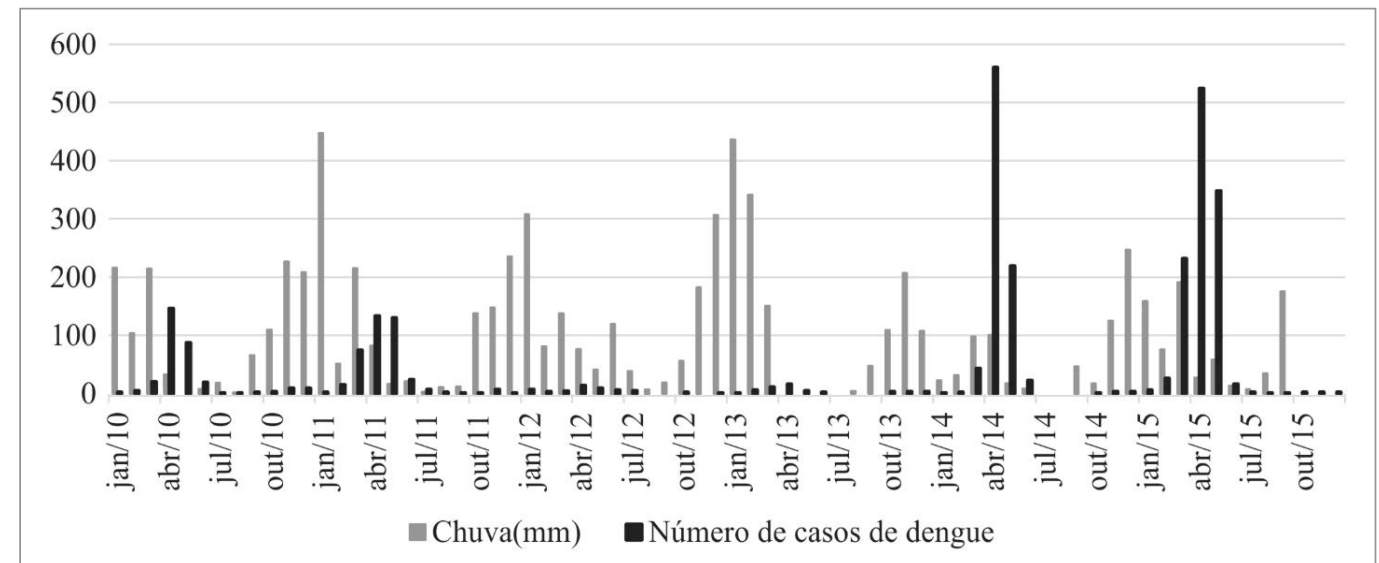

Fonte: Autores 
Considerando cada um dos anos de 2010 a 2015, na Figura 3 estão apresentados os mapas da área urbana de Três Corações, Minas Gerais, com cada ponto representando um caso de dengue, e as intensidades das ocorrências estimadas pelo método Kernel. A escala para a intensidade é dada em número de casos por $\mathrm{km}^{2}$. Pode-se observar nos mapas que as maiores intensidades de casos de dengue tendem a ocupar a Região Central e decrescem na direção das áreas mais afastadas.

Figura 3 - Distribuição espacial das ocorrências de dengue em Três Corações - MG, entre 2010 e 2015 e os respectivos mapas de intensidade $\left(\mathrm{em}\right.$ casos por $\mathrm{km}^{2}$ ), estimadas pelo método Kernel.

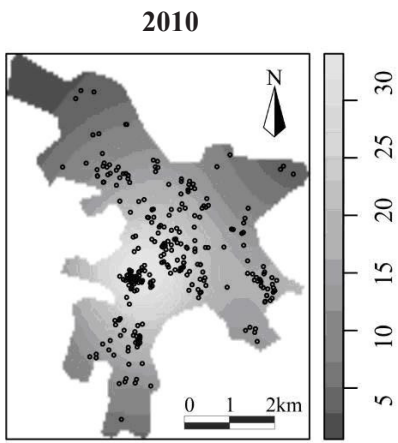

2013

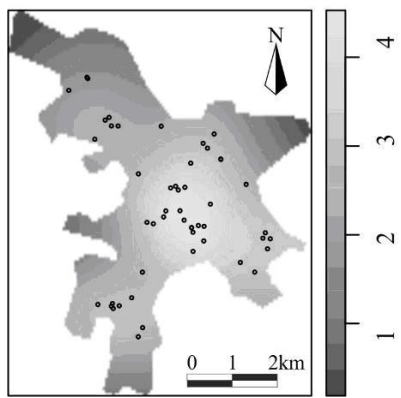

Fonte: Autores
2011

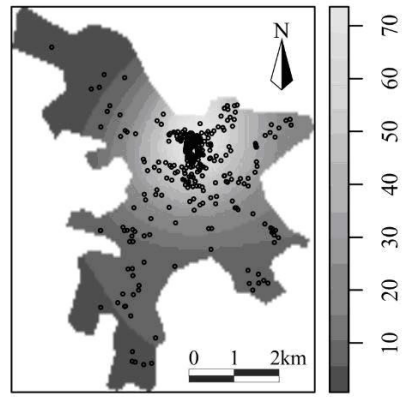

2014

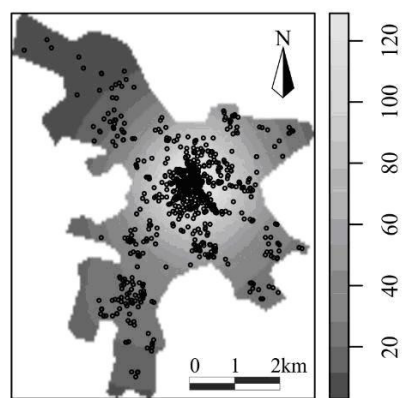

2012

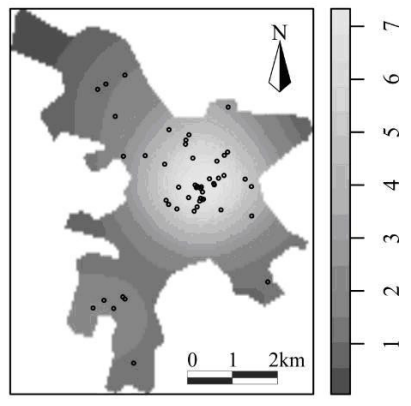

2015

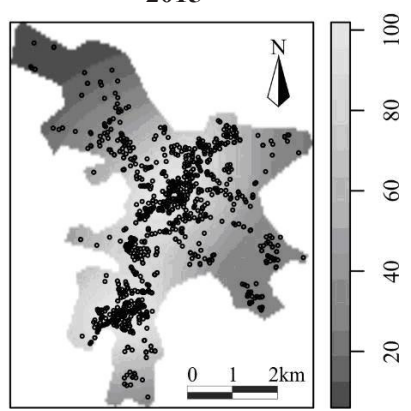

Na Figura 4 (A) está apresentado o resultado foi rejeitada ao nível de $5 \%$ de significância a da análise utilizando a estatística de teste $D$ baseada na função $K$. A partir do procedimento de Monte Carlo, o valor-p obtido foi igual a 0,001 , portanto, hipótese de que os casos de dengue apresentaram uma configuração completamente aleatória no tempo e no espaço.

Figura 4 - (A) Distribuição empírica da estatística $D$ obtida pelo procedimento de Monte Carlo e o valor da estatística calculada para os dados. (B) Gráfico da diferença entre a função $K$ estimada e o esperado para o caso de completa aleatoriedade espaço-temporal.

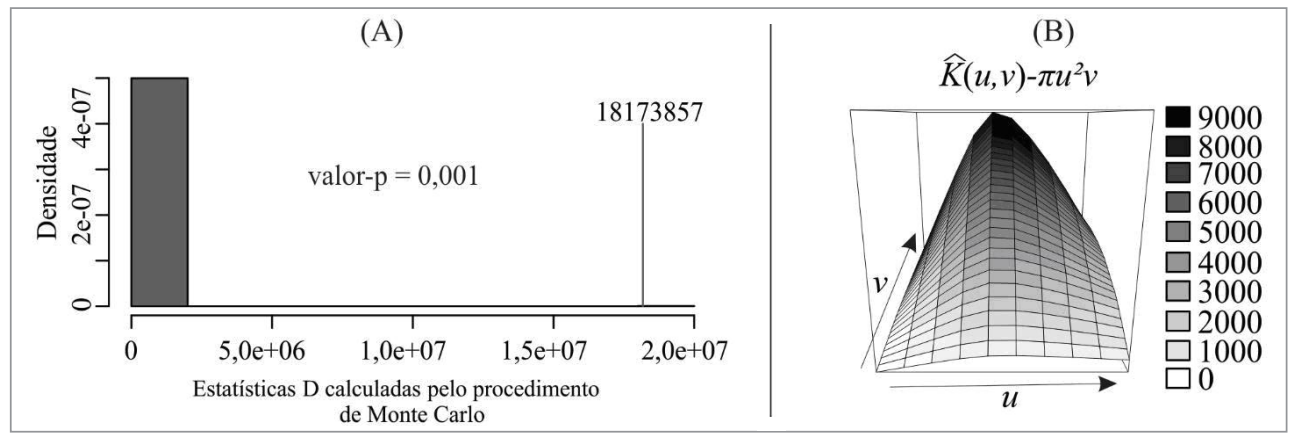

Fonte: Autores 
Ao executar a função $K$ e compará-la ao que seria esperado pela completa aleatoriedade espaçotemporal, o gráfico dessa análise, exposto na Figura 4 (B), mostra que houve um excesso de valores positivos, indicando, assim, que o comportamento das ocorrências de dengue em Três Corações exibiu um padrão de dependência, com agrupamentos de casos no espaço-tempo.

A análise utilizando o método estatístico de varredura localizou seis agrupamentos locais estatisticamente significativos, considerando um nível de significância de 5\%, como mostrado na Figura 5. Isso significa que, de todos os possíveis agrupamentos de casos dentro da região de estudo, no período de $1^{\circ}$ de janeiro de 2010 a 31 de dezembro de 2015, esses seis agrupamentos rejeitaram a hipótese de terem ocorrido de modo completamente aleatório, indicando, desta forma, algum tipo de interação entre os casos de dengue em cada um deles.

Figura 5 - Agrupamentos espaço-temporais das ocorrências de dengue encontrados pelo método de varredura com o software SaTScan ${ }^{\mathrm{TM}}$.

\begin{tabular}{|c|c|c|c|c|}
\hline & Agrup. & $\mathrm{N}^{\circ}$ de casos & Período & Valor-p \\
\hline & 1 & 61 & $08 / 02 / 2010$ a $26 / 01 / 2011$ & $1,0 \times 10^{-17}$ \\
\hline & 2 & 31 & $27 / 03 / 2010$ a $16 / 02 / 2011$ & 0,0032 \\
\hline & 3 & 163 & 02/03/2011 a 14/06/2011 & $1,0 \times 10^{-17}$ \\
\hline & 4 & 358 & $17 / 02 / 2014$ a $22 / 05 / 2014$ & $1,0 \times 10^{-17}$ \\
\hline . & 5 & 310 & $12 / 02 / 2015$ a $22 / 05 / 2015$ & $3,3 \times 10^{-17}$ \\
\hline $\begin{array}{llll}0 & 0 & 1 & 2 \mathrm{~km}\end{array}$ & 6 & 46 & $31 / 03 / 2015$ a $26 / 04 / 2015$ & 0,0017 \\
\hline
\end{tabular}

Fonte: Autores

Pode-se perceber na Figura 5 que os focos dos agrupamentos estão em locais distintos e com intervalos de tempo com diferentes comprimentos. Os agrupamentos 1 e 2, com 61 casos e 31 casos, respectivamente, foram detectados em intervalos de quase um ano, indicando que na região onde os casos ocorreram havia um foco em que as infecções pela dengue persistiram durante todo o período em questão.

Nos anos de 2012 e 2013, em que a quantidade de casos foi relativamente pequena se comparada com os outros anos, não foi detectado nenhum agrupamento espaço-temporal. Os dois maiores agrupamentos (4 e 5) foram registrados em intervalos de tempo semelhantes, ocorrendo no período de fevereiro a maio nos anos de 2014 e 2015, respectivamente. Deve-se observar que é neste período onde está concentrada a maioria dos casos nesses anos.
Diferentemente dos agrupamentos 1 e 2, os casos de dengue dos agrupamentos 3, 4, 5 e 6 ocorreram em um curto intervalo de tempo, caracterizando assim um surto de dengue para essas regiões naquele período.

\section{Discussão}

Estudos apontam que a dinâmica de ocorrência da dengue está fortemente relacionada a fatores climáticos. Viana e Ignotti ${ }^{(30)}$ constataram que, no Brasil, o período de maior incidência da dengue ocorre, em geral, nos períodos de maior intensidade das chuvas e com temperaturas mais altas. Já nos períodos mais frios e secos esse número diminui. Segundo Neiva e Cardoso, ${ }^{(31)}$ a presença de chuvas em determinada região pode influenciar o acúmulo de água parada em determinados locais, contribuindo para a procriação dos mosquitos 
transmissores. No entanto, os autores ressaltam que chuvas em excesso também podem levar a uma queda no número de casos da doença, uma vez que grandes quantidades de chuva podem inibir a capacidade de dispersão do mosquito e, consequentemente, diminuir sua reprodução. Silva et al. ${ }^{(32)}$ também apontam, por outro lado, que após dois ou três meses de estação seca, em função da baixa umidade relativa e a ausência de criadouros temporários, o meio se torna desfavorável à sobrevivência dos mosquitos, indicando que o final do período chuvoso fornece ambientes propícios para a proliferação do mosquito Aedes aegypti.

Esse comportamento foi observado para a cidade de Três Corações. Verificou-se que os períodos de grande incidência da doença (entre abril e junho) foram precedidos por períodos de chuva, como mostrado na Figura 2. Especificamente para os anos de 2014 e 2015, observou-se períodos moderados de chuvas em relação aos anos anteriores, isto é, um total pluviométrico médio mensal abaixo dos $200 \mathrm{~mm}$. Desta forma, volumes moderados de chuvas podem ter contribuído para o acúmulo de água parada em reservatórios, tornando o ambiente favorável para a proliferação do mosquito. Consequentemente, um aumento de casos notificados da doença veio a ocorrer, causando um surto epidêmico da doença no município. Em contrapartida, o município presenciou um grande volume de chuvas entre os anos de 2010 e 2013 que se intensificou, principalmente, em 2012 e 2013, atingindo um total pluviométrico médio mensal acima dos 200 $\mathrm{mm}$. No entanto, um comportamento inverso é visto em relação à frequência de casos notificados de dengue, que nos dois anos iniciais apresentou uma quantidade moderada, mas que diminuiu nos dois anos seguintes.

No que se refere à distribuição espacial das ocorrências de dengue, conforme exibido na Figura 2, a maior intensidade tende a ocupar a Região Central e decresce na direção das áreas mais afastadas. Uma razão para isso poderia ser em função da densidade populacional da cidade de Três Corações ter esse comportamento de ser maior no Centro da cidade e decrescer na direção das áreas periféricas.

A partir dos mapas na Figura 2, observa-se que a densidade populacional parece exercer papel fundamental para explicar as epidemias de dengue, haja vista que as regiões com maiores notificações de casos são as que apresentam as maiores concentrações de habitantes. A respeito disso, Cabral e Freitas ${ }^{(33)}$ apontam que um número maior de indivíduos, por $\mathrm{km}^{2}$, em uma região contribui para uma maior chance de contato com o vetor, ao encontrar grupos maiores de suscetíveis. Outra explicação é que regiões com altas densidades demográficas favorecem o aumento de produção de resíduos não orgânicos como latas, garrafas e sacos plásticos que cria condições propícias à proliferação do mosquito transmissor da dengue. O papel da influência da densidade demográfica no padrão de transmissão da dengue também pode ser encontrado nos estudos de Simone et al. ${ }^{(34)}$ e Machiner et al., ${ }^{(35)}$ entre outros. Portanto, os resultados deste estudo sugerem que as políticas públicas de combate à proliferação da dengue devem ser direcionadas para as áreas de maior densidade populacional.

Em relação à análise com a função $K$, os resultados obtidos indicaram um padrão de agrupamento espaço-temporal das ocorrências da dengue devido a um efeito de dependência entre as ocorrências, ou seja, a ocorrência de um caso aumenta a probabilidade de se ter novos casos no seu entorno em dias próximos. No entanto, esse efeito de agrupamento pode se dar também pelo fato de que muitos casos ocorreram em locais onde há um número maior de pessoas residindo, ou seja, um efeito de agrupamento captado por variações no valor médio do processo pontual, de modo que a intensidade espacial e a intensidade temporal variaram na região dentro do período em estudo (possivelmente devido à densidade populacional $\mathrm{e}$ aos períodos de chuva respectivamente).

Deve-se observar que os resultados obtidos no presente trabalho se referem a uma análise retrospectiva, indicando apenas um possível padrão 
de comportamento espaço-temporal da dengue. Esses resultados não significam, necessariamente, que um padrão espaço-temporal semelhante venha a ocorrer em tempos futuros, mas podem ser utilizados como uma ferramenta para que os agentes da saúde/vigilância epidemiológica possam investigar o que existe nessas localidades e períodos do ano que poderia potencializar a proliferação do mosquito transmissor, e traçar estratégias que auxiliem na prevenção e combate à dengue.

Finalmente, deve ser ressaltado que apesar deste trabalho focar na detecção de agrupamentos de casos da dengue, os métodos apresentados podem ser utilizados para caracterizar a distribuição espaço-temporal de outras epidemias, tais como chikungunya, febre amarela urbana, zica etc. Esses métodos podem ainda ser aplicados para analisar a distribuição de doenças epidêmicas ou pandêmicas ainda pouco conhecidas, como é o caso da Covid-19, o que pode contribuir para a tomada de decisão sobre a prevenção e combate dessas doenças.

\section{Conclusão}

Os métodos estatísticos baseados na teoria de processos pontuais permitiram caracterizar a distribuição espaço-temporal dos casos da dengue na região da cidade de Três Corações, Minas Gerais, detectando que no final dos períodos mais chuvosos as pessoas que vivem em regiões mais densamente povoadas estão mais propensas a contrair a doença. Ocorrências futuras podem apresentar um padrão semelhante ao observado nesse trabalho; no entanto, os resultados obtidos se referem a uma análise retrospectiva, indicando apenas um possível padrão de comportamento espaço-temporal da dengue. Os métodos utilizados não podem ser usados para previsão de casos futuros, mas os resultados podem ser usados como parâmetros pelos agentes da saúde/vigilância epidemiológica, no que se refere à tomada de decisão sobre estratégias que auxiliem na prevenção e combate à dengue.

\section{Agradecimentos}

À Coordenação de Aperfeiçoamento de Pessoal de Nível Superior (CAPES), pelo auxílio financeiro durante a pesquisa e à Vigilância Epidemiológica da cidade de Três Corações, pelos dados cedidos.

\section{Referências}

1 Ministério da Saúde (BR). Guia de vigilância epidemiológica [Internet]. 2005 [citado 2020 maio 5]. Disponível em: http://bvsms.saude. gov.br/bvs/publicacoes/Guia_Vig_Epid_novo2. pdf

2 Mendonça FA, Souza AV, Dutra DA. Saúde pública, urbanização e dengue no Brasil. Soc Nat. 2009;21(3):257-69.

3 Valle D, Bellinato DF, Viana-Medeiros PF, Lima JBP, Martins Junior ADJ. Resistance to temephos and deltamethrin in Aedes aegypti from Brazil between 1985 and 2017. Mem Inst Oswaldo Cruz. 2019;114.

4 World Health Organization. Dengue guidelines for diagnosis, treatment, prevention and control. Geneva: WHO; 2009.

5 Ministério da Saúde (BR). Guia de vigilância em saúde: volume 2 [Internet]. 2017 [citado 2020 maio 8]. Disponível em: https://bvsms. saude.gov.br/bvs/publicacoes/guia_vigilancia_ saude_volume_2.pdf

6 Instituto Oswaldo Cruz. Aedes e dengue: vetor e doença [Internet]. 2016 [citado 2016 nov 14]. Disponível em: http://www.ioc.fiocruz.br/ dengue/textos/aedesvetoredoenca.html

7 Dias LB, Almeida SC, Haes TM, Mota LM, Roriz-Filho JS. Dengue: transmissão, aspectos clínicos, diagnóstico e tratamento. Medicina (Ribeirão Preto). 2010;43(2):143-52.

8 World Health Organization - WHO. Frequently asked questions on dengue [Internet]. 2016 [cited 2016 Nov 14]. Available from: http://www.wpro. who.int/mediacentre/releases/2010/20100916 FAQs/en/ 
9 Higa Y. Dengue vectors and their spatial distribution. Trop Med Health [Internet]. 2011 [cited 2020 May 8];39:17-27. Available from: https://www.jstage.jst.go.jp/article/ tmh/39/4SUPPLEMENT/39_2011-S04/_pdf/char/en

10 Warkentien T, Pavlicek R. Dengue fever: historical perspective and the global response. J Infect Dis Epidemiol [Internet]. 2016 [cited 2020 May 8];2(2). Available from: https:// clinmedjournals.org/articles/jide/journalof-infectious-diseases-and-epidemiologyjide-2-015.pdf

11 Ministério da Saúde (BR). SINAN: Sistema de Informação de Agravos de Notificação [Internet]. 2020 [citado 2020 mar 12]. Disponível em: http://portalsinan.saude.gov.br/

12 Ferreira LM. Mapeamento dos casos de dengue na cidade de Lavras-MG, no período de 20072010 [dissertação]. Lavras: Universidade Federal de Lavras; 2012.

13 Toan DTT, Hu W, Quang TP, Hoat LN, Wright P, Martens P. Hot spot detection and spatiotemporal dispersion of dengue fever in Hanoi, Vietnam. Glob Health Action. 2013;6(1).

14 Alzahrani AG, Mazroa MAA, Alrabeah AM, Ibrahim AM, Mokdad AH, Memish ZA. Geographical distribution and spatio-temporal patterns of dengue cases in Jeddah governorate from 2006-2008. Trans R Soc Trop Med Hyg. 2012;107:23-9.

15 Malik A, Yasar A, Tabinda AB, Zaheer IE, Malik K, Batool A, et al. Assessing spatiotemporal trend of vector breeding and dengue fever incidence in association with meteorological conditions. Environ Monit Assess. 2017;189(4).

16 Silva FG, Magalhães SCM. Correlação entre fatores climáticos, socioambientais e a dengue na microrregião Montes Claros/MG. Caminhos de Geografia. 2017;18(61): 231-44.

17 Prefeitura Municipal de Três Corações - MG. Estatísticas [Internet]. 2020 [citado 2020 maio 6]. Disponível em: https://www.trescoracoes. mg.gov.br/index.php/2014-12-30-11-1009/5190-estatisticas
18 Instituto Brasileiro de Geografia e Estatística. Três Corações [Internet]. 2020 [citado 2020 jan 15]. Disponível em: https://cidades.ibge. gov.br/brasil/mg/tres-coracoes

19 Climate-data.org. Clima Três Corações (MG) [Internet]. 2020 [citado 2020 maio 6]. Disponível em: https://pt.climate-data.org/ america-do-sul/brasil/minas-gerais/trescoracoes- 25070

20 Google INC. Google Earth [software]. Versão 7.1.5.1557. 2017. Disponível em: https://www. google.com.br/intl/pt-BR/earth/

21 Loecher M, Ropkins K. RgoogleMaps and loa: unleashing $\mathrm{R}$ graphics power on map tiles. J Stat Soft. 2015;63(4):1-18.

22 Pebesma EJ, Bivand RS. Classes and methods for spatial data: the sp Package [Internet]. 2005 [cited 2020 May 6]. Available from: https:// cran.r-project.org/web/packages/sp/vignettes/ intro_sp.pdf

23 R Core Team. R: a language and environment for statistical computing. Vienna: The R Foundation for Statistical Computing; 2017.

24 Schabenberger O, Gotway CA. Statistical methods for spatial data analysis. Boca Raton: CRC Press; 2017.

25 Diggle PJ, Chetwynd AG, Haggkivist R, Morris SE. Second order analysis of spacetime clustering. Stat Methods Med Res. 1995;4(2):124-36.

26 Gabriel E, Rowlingson B, Diggle PJ. stpp: an R package for plotting, simulating and analyzing spatio-temporal point patterns. J Stat Soft. 2013;53(2):1-29.

27 Diggle PJ. Statistical analysis of spatial and spatio-temporal point patterns. $3^{\text {rd }}$ ed. Boca Raton: CRC Press; 2013.

28 Kulldorff M, Heffernan R, Hartman J, Assunção R, Mostashari F. A space-time permutation scan statistic for disease outbreak detection. PLoS Med. 2005;2(3).

29 Kulldorff M, Information Management Services Inc. SaTScan ${ }^{\mathrm{TM}}$ [software]. Version 9. 1.1. 2013. 
30 Viana DV, Ignotti E. A ocorrência da dengue e variações meteorológicas no Brasil: revisão sistemática. Rev Bras Epidemiol. 2013;16(2): 240-56.

31 Neiva HS, Cardoso C. Clima e dengue: uma ajuda para compreender a epidemia na cidade do Rio de Janeiro. Rev Pol Publ [Internet]. 2018 [citado 2020 maio 8]; 22:1135-50. Disponível em: http://www.redalyc.org/ articulo.oa? $\mathrm{id}=321158844058$

32 Silva JS, Mariano ZF, Scopel I. A influência do clima urbano na proliferação do mosquito Aedes aegypti em Jataí (GO), na perspectiva da Geografia Médica. Hygeia. 2007;3(5).

33 Cabral JA, Freitas MV. Distribuição espacial e determinantes socioeconômicos e demográficos da dengue nos municípios brasileiros. Rev Bras Estud Reg Urbanos. 2012;6(1):8195.

34 Simone TS, Nogueira RMR, Araújo ESM. Dengue virus surveillance: the co-circulation of DENV-1, DENV-2 and DENV-3 in the state of Rio de Janeiro, Brazil. Trans R Soc Trop Med Hyg. 2004;98:553-62.

35 Machiner F, Rodrigues DJ, Andrade EA. Distribuição de dengue no norte de Mato Grosso, Brasil, 2001-2005. Cad Saúde Colet. 2009; 17(3):627-40. 